\title{
Uterine rupture at 33 weeks following previous B-lynch suture
}

\section{S Amarasekara ${ }^{1}$, D Dissanayake ${ }^{2}$, A Jayawardana ${ }^{2}$, D Silva ${ }^{1}$}

Ceylon Medical Journal 2011; 56: 121-123

\section{Case report}

A 31-year old woman in her third pregnancy with one previous lower segmental caesarian section (LSCS) and one first trimester miscarriage came to the antenatal ward at 33 weeks gestation with a 24 hour history of lower abdominal pain. In 2004 her first delivery required an emergency LSCS due to lack of progression. She subsequently developed severe primary post partum haemorrhage $(\mathrm{PPH})$ due to uterine atony. Medical management with uterotonics failed and B-Lynch

${ }^{1}$ Department of Obstetrics and Gynaecology, University of Sri Jayawardenapura, Sri Lanka, ${ }^{2}$ Colombo South Teaching Hospital, Sri Lanka.

Correspondence: SA, e-mail: <shamila@doctors.org.uk>. Received 22 November 2010 and revised version accepted 25 March 2011. Competing interests: none declared. 
compression sutures were applied using No. 1 chromic catgut.

In the present pregnancy her antenatal course had been uneventful. There was no antecedent history of trauma before admission. There was no vaginal bleeding or dribbling and the fetal movements were satisfactory. She was not pale and her abdomen was soft with no palpable uterine contractions or scar tenderness. The symphyseal-fundal height was $35 \mathrm{~cm}$ with a breech presentation. Speculum and vaginal examinations revealed that she was not in active labour.

Cardiotocogram (CTG) was reassuring. Ultrasound examination showed a single live fetus in breech presentation and fetal growth was compatible with the period of gestation. Her urine microscopy and full blood count were normal. She was admitted for conservative management and her abdominal pain responded to mild analgesics.

Late in the evening on day 2 of her hospital stay she complained of severe abdominal pain and on examination she was pale, tachycardic and hypotensive. Her abdomen was very tender and tense but no vaginal bleeding was detected. A bedside ultrasound examination showed a large amount of free fluid in the peritoneal cavity with a well-contracted uterus and a dead fetus lying outside, within the peritoneal cavity. The probable diagnosis of uterine rupture was made and the woman was prepared for an emergency laparotomy with consent obtained for hysterectomy.

During laparotomy a haemoperitoneum of about 21 was found. A large uterine rupture at the fundus along the coronal plane towards both cornua and also extending posteriorly in the midline was identified as the source of the profuse bleeding (Figure 1). The previous uterine scar was intact. Haemostasis was achieved by repairing the defect in two layers with number one polyglactin (Figure 2).

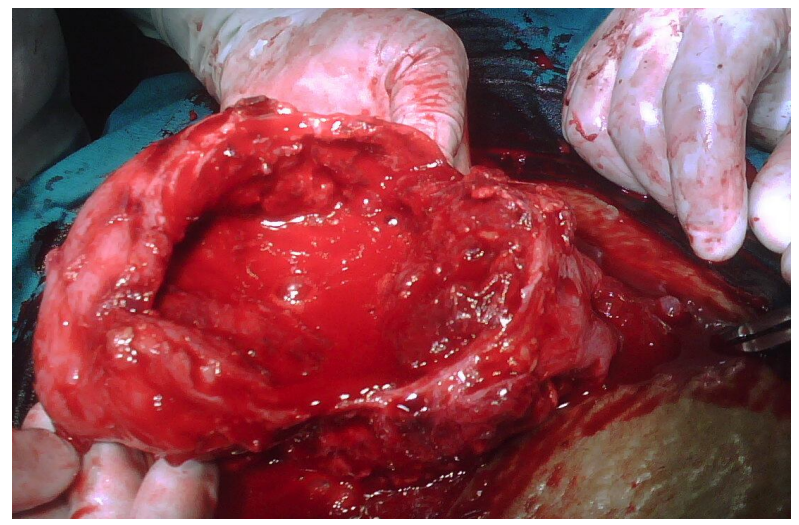

Figure 1. Large uterine rupture at the fundus extending along the coronal plane.



Figure 2. Uterus after repairing defect.

Four units of blood, two units of fresh frozen plasma and one unit of cryoprecipitate were administered perioperatively. She was observed in the intensive care unit for 24 hours and received broad-spectrum intravenous antibiotics and thromboprophylaxis for five days. The woman was sent home on 7 th postoperative day with counseling regarding the risk of early uterine rupture in future pregnancies and contraceptive.

\section{Discussion}

Post partum haemorrhage (PPH) remains one of the major causes of maternal death in both developed and developing countries and accounts for $25 \%$ of maternal mortality worldwide [1]. In Sri Lanka it has been the leading direct cause of maternal mortality for the past decade [2].

The clinician has a choice of conservative options to control PPH, including pharmacological and mechanical methods. The last option available is a hysterectomy. This is associated with high morbidity and loss of fertility. In order to avoid hysterectomy, several authors have introduced compressive uterine suture techniques into obstetric practice [3-6]. The most widely used of these being the B-Lynch and modified B-Lynch brace sutures and several case series have been published describing their success.

However, there have also been recent reports of complications due to these techniques including partial ischaemic necrosis after a B-Lynch procedure needing hysterectomy, a case of pyometria following a uterine brace suture and erosion of a B-Lynch suture through the uterine wall with the stitch identified protruding from the cervical os [7, 8, 9]. To date this is only the second reported incident of uterine rupture during a subsequent pregnancy following the placement of B- Lynch sutures [10].

The damage caused by the compression sutures occurs in the immediate post-operative period and is related to the degree of tension and ischaemia exerted by the suture on the myometrium [7]. The type of suture material may also play a role [10]. Although No. 2 chromic 
catgut suture was B-Lynch's choice, materials such as polyglactin have been used subsequently [4]. However, no randomised studies have been carried out to assess the correct choice of suture material.

The authors recommend that women who receive uterine brace sutures be counselled post-operatively regarding possible risks to further pregnancies and have long term follow up to detect and prevent potential complications. As more and more clinicians carry out brace sutures for the treatment of PPH it is inevitable that complications will ensue. As suggested previously perhaps a Sri Lankan national data base can be maintained to register all women who receive brace sutures to ensure proper follow up and reporting of complications [10].

\section{References}

1. World Health Organization. Maternal mortality in 2005: estimates developed by WHO, UNICEF, UNFPA, and the World Bank. 2007, World Health Organization.

2. Unpublished Data, Family Health Bureau, Sri Lanka.

3. B-Lynch C, Coker A, Lawal AH, et al. The B-Lynch surgical technique for the control of massive postpartum haemorrhage: an alternative to hysterectomy? Five cases reported. British Journal of Obstetrics and Gynaecology 1997; 104: 372-5.

4. Hayman RG, Arulkumaran S, Steer PJ. Uterine compression sutures: surgical management of postpartum hemorrhage. Obstetrics and Gynecology 2002; 99: 502-6.

5. Cho JH, Jun HS, Lee CN. Hemostatic suturing technique for uterine bleeding during cesarean delivery. Obstetrics and Gynecology 2000; 96: 129-31.

6. Tjalma WA, Jacquemyn Y. A uterus-saving procedure for postpartum hemorrhage. International Journal of Gynaecology and Obstetrics 2004; 86: 396-7.

7. Joshi VM, Shrivastava M. Partial ischemic necrosis of the uterus following a uterine brace compression suture. British Journal of Obstetrics and Gynaecology 2004; 111: 279- 80.

8. Ochoa M, Allaire AD, Stitely ML. Pyometria after hemostatic square suture technique. Obstetrics and Gynecology 2002; 99: 506-9.

9. Grotegut CA, Larsen FW, Jones MR, Livingston E. Erosion of a B-Lynch suture through the uterine wall: a case report. Journal of Reproductive Medicine 2004; 49: 849-52.

10. Pechtor K, Richards B, Paterson H. Antenatal catastrophic uterine rupture at 32 weeks of gestation after previous BLynch suture. British Journal of Obstetrics and Gynaecology 2010; 117: 889-91. 